\title{
Crescimento e teor de óleo essencial de mentas com diferentes concen- trações de potássio na solução nutritiva ${ }^{1}$
}

\author{
Tânea MB Garlet²; Osmar S Santos³; Sandro LP Medeiros³; Paulo A Manfron³; Danton C Garcia³; Sílvia \\ S Sinchak ${ }^{4}$
}

${ }^{2}$ UNICRUZ, Andrade Neves 308, 98025-810 Cruz Alta-RS; taneagarlet@hotmail.com; ${ }^{3}$ UFSM, 97105-900 Santa Maria-RS; ${ }^{4}$ Estudante de Agronomia UFSM.

\section{RESUMO}

O trabalho foi conduzido em casa de vegetação da UFSM, RS, de outubro a dezembro de 2004. Foram conduzidos três experimentos simultâneos, cada um com uma espécie de Mentha (M.arvensis, $M$. x gracilis e $M$. x piperita var. citrata) em quatro soluções hidropônicas com diferentes concentrações de K (276; 414; 552 e $\left.690 \mathrm{mg} \mathrm{L}^{-1}\right)$ e idades das plantas. O delineamento experimental utilizado foi de blocos ao acaso, com cinco repetições, tendo os tratamentos fatorial para cada espécie: 4x6, 4x7 e 4x8, sendo quatro concentrações de $\mathrm{K}$ e idades da planta determinadas semanalmente a partir dos 27 dias. Foram avaliadas a altura das plantas; área foliar; fitomassa fresca e seca de folhas, hastes; relação folha:haste; e teor de óleo essencial em folhas frescas. O aumento na concentração de $\mathrm{K}$ nas soluções hidropônicas afeta negativamente o crescimento e a acumulação de fitomassa em plantas de Mentha, porém proporciona aumento no teor de óleo essencial por planta. Em todas as colheitas consecutivas observou-se aumento no crescimento das plantas das três espécies. As plantas de $M$. arvensis e M. x piperita var. citrata acumularam mais fitomassa de hastes do que de folhas, o contrário ocorrendo com M. x gracilis que, além disso, apresentou maior produção equivalente de óleo por hectare, na concentração mais baixa de K. As concentrações de K sugeridas para favorecer o crescimento de Mentha em cultivo hidropônico encontram-se na faixa entre 276 e $414 \mathrm{mg} \mathrm{L}^{-1}$.

Palavras-chave: Mentha arvensis, Mentha $x$ gracilis, Mentha $\mathrm{x}$ piperita var. citrata, óleo aromático, produtividade.

\section{ABSTRACT}

Growth and essential oil content of mints at different potassium concentrations in the nutrient solution

The work was carried out at the Universidade Federal de Santa Maria, Rio Grande do Sul State, Brazil, from October to December 2004. Three simultaneous experiments were carried out, each one with one Mentha species (M. arvensis, M. x gracilis and M. x piperita var. citrata) in four hydroponic solutions with different $\mathrm{K}$ concentrations (276; 414; 552 and $\left.690 \mathrm{mg} \mathrm{L}^{-1}\right)$ and plant ages. The experimental design was of randomized complete blocks, with five replications, the treatments showing a factorial for each species: $4 \times 6$, $4 \times 7$ and $4 \times 8$, four $\mathrm{K}$ concentrations and plant ages being determined weekly starting 27 days after planting date. The height of plants; foliar area; fresh and dry phytomass of leaves and stems; leaf:stem relation; and the content of essential oil in fresh leaves were evaluated. The increase of $\mathrm{K}$ concentrations in the hydroponic solutions affected negatively the growth and accumulation of phytomass in mint plants, but allowed an increase in the content of essential oil per plant. In all consecutive harvesting dates an increase in the growth of the three species of plants were observed. M. arvensis and M. x piperita var. citrata accumulated more stem than leaf phytomass, the reverse occurring with $M$. $x$ gracilis, which also presented a higher equivalent production of oil per ha, in the lowest $\mathrm{K}$ concentration. A good growth of Mentha under hydroponic cultivation was observed under $\mathrm{K}$ concentrations of 276 and $414 \mathrm{mg} \mathrm{L}^{-1}$.

Keywords: Mentha arvensis, Mentha $x$ gracilis, Mentha x piperita var. citrata, yield, aromatic oil.

(Recebido para publicação em 11 de julho de 2006; aceito em 22 de maio de 2007)

$\mathrm{C}$ om sabor e aroma refrescantes, as mentas ou hortelãs destacam-se pelo uso culinário ou em chás medicinais, para combater parasitas intestinais e distúrbios digestivos (Lorenzi \& Matos, 2002). As glândulas oleíferas, principalmente das folhas, concentram óleos voláteis ricos em terpenóides de amplo interesse industrial, em produtos farmacêuticos, alimentícios, cosméticos e aromatizantes do tabaco.

As mentas são plantas muito exigentes em fertilidade e irrigação (Maia, 1998a), o manejo nutricional é determinante na obtenção de matériaprima de qualidade. O cultivo em hidroponia vem despertando interesse como uma forma de produzir material vegetal de qualidade, com alta produtividade quando comparado ao sistema tradicional de cultivo no solo (Castellane \& Araújo, 1995). Mairapetyan (1999) afirma que plantas aromáticas, produzidas em sistema hidropônico apresentam elevada produtividade e três a seis vezes mais óleo essencial por planta do que em sistemas convencionais de cultivo. A hidroponia permite ainda reduzir a duração do ciclo produtivo com reflexos na produtividade (Santos, 2000). A proporção entre os nutrientes da solução nutritiva afeta tanto o crescimento como a produção de biomassa e o teor de óleo essencial em Mentha (Brown et al., 2003).

O potássio (K) é, geralmente, o nutriente mais abundante em todas as culturas. Está presente nos tecidos em sua maior parte na forma solúvel em água (Malavolta, 2006). Trata-se de elemento essencial com ação fisiológica, como: extensão celular; abertura dos

${ }^{1}$ Parte da Tese de doutorado apresentada pelo primeiro autor à UFSM. 
estômatos; transporte no floema; compensação de cargas; ativador de enzimas como a ATPase, sintetases de amido e de proteínas; na resistência aos estresses em geral como seca, salinidade, baixas temperaturas e na resistência às doenças (Marschner, 1995).

$\mathrm{O}$ íon $\mathrm{K}^{+}$tem intensa mobilidade no xilema e floema, possibilitando a regulação do balanço interno, desde que sua absorção seja eficiente (Bataglia, 2005). A demanda de K para o crescimento das plantas é elevada, variando de 10 a $60 \mathrm{~g} \mathrm{~kg}^{-1}$ de matéria seca. Se sua disponibilidade é baixa, o crescimento da planta é retardado e a retranslocação líquida ou a remobilização deste nutriente das folhas maduras e caule é aumentada. Sob deficiência severa, esses órgãos tornam-se cloróticos e necróticos (Marschner \& Cakmak, 1989). Por outro lado, o excesso de $\mathrm{K}$ raramente é observado, exceto nos casos de danos por sais resultantes de aplicação excessiva de $\mathrm{KCl}$, podendo provocar efeitos adversos de toxicidade, diminuindo o crescimento ou a produção da planta (Bataglia, 2005).

Os estudos de produção de plantas medicinais em hidroponia ainda são escassos. Este trabalho objetivou avaliar o crescimento e o teor de óleo essencial em três espécies de Mentha, submetidas a diferentes concentrações de $\mathrm{K}$ na solução nutritiva e em diferentes idades das plantas.

\section{MATERIAL E MÉTODOS}

Os experimentos foram realizados de outubro a dezembro de 2004, em casa de vegetação de $250 \mathrm{~m}^{2}$ coberta com polivinilclorídrico (PVC), com $200 \mu \mathrm{m}$ de espessura, em área experimental da Universidade Federal de Santa Maria, RS.

Foram realizados três experimentos simultâneos, no delineamento em blocos ao acaso, com cinco repetições, tendo os tratamentos fatorial para cada espécie: 4x6 (Mentha arvensis L.), 4x7 (Mentha $x$ gracilis Sole) e 4x8 (Mentha $\mathrm{x}$ piperita var. citrata (Ehrh.) Briq.), sendo quatro concentrações de K de 276 , 414, 552 e $690 \mathrm{mg} \mathrm{L}^{-1}$ e idades de colheita das plantas em dias de 27; 34; 41;
48; 55 e 62 em M. arvensis; 27; 34; 41; 48; 55; 62 e 69 em M. gracilis; 27; 34; 41; 48; 55; 62; 69 e 76 em $M$. x piperita var.citrata.

As mudas das espécies de Mentha foram obtidas a partir de matrizes produzidas em solo, em laboratório da UNICRUZ, e preparadas a partir de estacas caulinares com $4 \mathrm{~cm}$ de comprimento e quatro folhas, postas para enraizar em espuma fenólica com dimensões de $2 \times 2 \times 2 \mathrm{~cm}$, conduzidas em berçário constituído por tubos de polipropileno, com $4 \mathrm{~m}$ de comprimento e $3 \mathrm{~cm}$ de largura, colocados sobre cavaletes com desnível de $2 \%$ para escoamento da solução nutritiva. As mudas permaneceram no berçário por 20 dias, quando apresentaram de oito a nove folhas desenvolvidas e foram transplantadas para as bancadas de produção final, constituídas por canais de cultivo de polipropileno com $6 \mathrm{~m}$ de comprimento por $10 \mathrm{~cm}$ de largura, sustentados por cavaletes de $0,80 \mathrm{~m}$ de altura, com declividade de $2 \%$. Utilizouse o espaçamento de $0,25 \mathrm{~m}$ nos canais e $0,40 \mathrm{~m}$ entre os canais, correspondendo à densidade de 10 plantas $\mathrm{m}^{-2}$.

Os experimentos foram conduzidos no sistema NFT (Técnica do Fluxo Laminar de Nutrientes), com a solução nutritiva distribuída nos canais de cultivo na vazão de $1,8 \mathrm{~L} \mathrm{~s}^{-1}$, através de conjunto de moto-bomba de 0,5 HP. A solução foi recolhida no final da bancada de cultivo através de calha coletora, retornando a um reservatório de PVC, com capacidade de $500 \mathrm{~L}$.

A solução nutritiva padrão foi fixada em $276 \mathrm{mg} \mathrm{L}^{-1}$, calculada a partir da quantidade de nutrientes extraídos por M. arvensis, obtidos por Paulus et al. (2004). Na produção de mudas, essa solução foi diluída a $50 \%$. Para o preparo da solução nutritiva utilizaram-se os seguintes nutrientes ( $\left.\mathrm{mg} \mathrm{L}^{-1}\right)$ : $\mathrm{K}=276$; $\mathrm{N}=211,4 ; \quad \mathrm{P}=30,35 ; \quad \mathrm{Ca}=71,34 ;$ $\mathrm{Mg}=32,56 ; \quad \mathrm{S}=40,72 ; \mathrm{Mn}=6,084 ;$ $\mathrm{Cu}=0,075 ; \quad \mathrm{Zn}=0,431 ; \quad \mathrm{B}=2,884 ;$ $\mathrm{Mo}=0,0883$; Fe-EDTA=0,5 L. A fonte de ferro foi obtida através da dissolução de $24,1 \mathrm{~g}$ de sulfato de ferro em 400 $\mathrm{mL}$ de água e 25,1 g de Na-EDTA em
$400 \mathrm{~mL}$ de água, conforme a metodologia descrita por Castellane \& Araújo (1995). Os micronutrientes foram fornecidos na forma de solução concentrada (cinco vezes) dos sais em 200 $\mathrm{mL}$ de água, completando-se o volume para $1 \mathrm{~L}$, após completa diluição. A partir da solução padrão variou-se a concentração de K para 414, 552 e 690 mg $\mathrm{L}^{-1}$ pela adição de 262,$4 ; 524,8$ e 787,2 $\mathrm{mg} \mathrm{L}^{-1}$ de $\mathrm{KCl}$, respectivamente.

A condutividade elétrica (CE) inicial das soluções com 276, 414, 552 e 690 $\mathrm{mg} \mathrm{L}^{-1}$ de $\mathrm{K}$ foi, respectivamente, 2,38; 2,72; 3,30 e 3,87 $\mathrm{mS} \mathrm{cm}^{-1}$. O controle foi realizado a cada dois dias, sendo feita a reposição de $50 \%$ dos nutrientes sempre que a $\mathrm{CE}$ atingia $50 \%$ do valor inicial. $\mathrm{O}$ pH foi corrigido a cada dois dias para o valor de 6,0 \pm 0,2 após completar o volume dos reservatórios com água. O controle da circulação das soluções foi efetuado com auxílio de temporizador programado para acionar a moto-bomba durante $15 \mathrm{~min}$, com intervalos de 15 minutos, no período diurno (07:00-20:00 h), e 15 min a cada intervalo de $2 \mathrm{~h}$ no período noturno.

Foram realizadas colheitas semanais, com a última efetuada para cada espécie em: 07 (M. arvensis ), 14 (M. x gracilis) e 21/12/04 (M. x piperita var. citrata). O ponto de colheita foi determinado pela floração conforme Duriyaprapan et al. (1986). Todavia, durante o período do experimento $M$. x piperita var. citrata não floresceu, fato este observado a campo apenas em março de 2005. As plantas coletadas foram separadas em raízes e parte aérea (hastes, folhas e flores). As flores foram acrescentadas junto às folhas devido à presença de óleo. As raízes não foram avaliadas por causa do entrelaçamento das plantas contíguas, inviabilizando sua correta separação. Das hastes e folhas determinou-se a fitomassa fresca. Após a pesagem das folhas retiraram-se discos foliares, com vazador de $0,02 \mathrm{dm}^{2}$ de área para determinação da área foliar. A seguir, acondicionaram-se as plantas em sacos de papel, levando-as à estufa de secagem com ventilação forçada de ar a $65^{\circ} \mathrm{C}$, até massa constante. Também foi determinada a altura das 
plantas e a relação folha:haste em fitomassa fresca e seca.

Amostras de $100 \mathrm{~g}$ de folhas frescas foram utilizadas para a extração do óleo essencial, em quatro repetições. O material foi acondicionado em sacos plásticos e armazenado em freezer $\left(-15^{\circ} \mathrm{C}\right)$ até o momento da extração. A técnica utilizada foi a hidrodestilação em aparelho de Clevenger, por 2 horas, em balão volumétrico de $2 \mathrm{~L}$. Os hidrolatos recolhidos com éter etílico foram levados em funil de separação e submetidos à secagem com sulfato de sódio anidro. O sal foi removido por filtração simples e o solvente evaporado em rotavapor. $\mathrm{O}$ óleo resultante foi pesado para determinação do teor e rendimento estimado por planta e por área.

Os dados obtidos foram submetidos à análise de variância e interpretados por meio da análise de regressão, pelo método dos polinômios ortogonais para os efeitos principais e suas interações, selecionando-se o componente de maior grau significativo. Utilizou-se o teste $\mathrm{F}$ a 1 e $5 \%$ de probabilidade para avaliação das equações polinomiais.

\section{RESULTADOS E DISCUSSÃO}

Nas três espécies de Mentha estudadas, a interação entre doses de potássio (K) e idades da planta foi significativa $(\mathrm{P}<0,05)$ para as variáveis: altura de planta, área foliar, fitomassa fresca e seca de folhas e de hastes. Em todas as colheitas observou-se incremento dessas variáveis. Para as mesmas, houve resposta quadrática de regressão em $M$. arvensis e em $M$. x piperita var. citrata, enquanto $M . x$ gracilis seguiu resposta linear. A relação folha:haste em fitomassa fresca e seca foi significativa somente para $M$. x gracilis.

A altura de planta em $M$. arvensis (Figura 1a) foi estatisticamente significativa em relação às concentrações de K para as idades a partir dos 48 dias, indicando que as doses estimadas de $\mathrm{K}$ para obtenção da altura máxima encontram-se entre 391,2 e 400,2 $\mathrm{mg} \mathrm{L}^{-1}$. O desempenho foi semelhante para $M$. x piperita var. citrata (Figura 2a), cuja significância ocorreu a partir dos 34 dias, com as melhores doses de $\mathrm{K}$ estimadas entre 350,6 e 416,0 $\mathrm{mg} \mathrm{L}^{-1}$. No entanto, $M$. $\mathrm{x}$ gracilis (Figura $3 \mathrm{a}$ ) mostrou significância dos 41 dias em diante, com a altura das plantas durante o período de desenvolvimento, apresentando-se mais baixa naquelas que foram submetidas à maior concentração de $\mathrm{K}$, evidenciando-se que concentrações de $\mathrm{K}$ acima de $276 \mathrm{mg} \mathrm{L}^{-1}$ influenciam negativamente o desenvolvimento dessa espécie, em decorrência do aumento na condutividade elétrica de 2,38 para $3,87 \mathrm{mS} \mathrm{cm}^{-1}$ nas soluções mais concentradas, com elevação na pressão osmótica que interfere na absorção de nutrientes.

Valmorbida (2003), ao cultivar $M$. piperita, encontrou altura máxima de $101,94 \mathrm{~cm}$ nas plantas submetidas aos níveis de potássio de 58,50/117,00 mg $\mathrm{L}^{-1}$ na solução hidropônica. Neste estudo, as maiores alturas do período experimentado correspondem a 122,50 ( $M$. arvensis), 97,62 (M. x gracilis) e 116,13 $\mathrm{cm}$ (M. x piperita var. citrata).

Os dados de área foliar foram significativos a partir dos 41 dias de idade para as três espécies de Mentha, com as doses de K estimadas para máxima área foliar em M. arvensis (Figura 1b) e em M. x piperita var. citrata (Figura $2 \mathrm{~b}$ ) variando de 398,3 a $417,8 \mathrm{mg} \mathrm{L}^{-1}$ e de 375,5 a 414,4 $\mathrm{mg} \mathrm{L}^{-1}$, respectivamente, cujos valores máximos na última colheita foram 77,83 $\mathrm{dm}^{2}$ e 97,74 $\mathrm{dm}^{2}$. A área foliar máxima de $M$. x gracilis (Figura 3b) aos 69 dias, na concentração de $\mathrm{K}$ de $276 \mathrm{mg} \mathrm{L}^{-1}$ foi de 79,05 $\mathrm{dm}^{2}$ apresentando redução de $40 \%$ com $690 \mathrm{mg} \mathrm{L}^{-1}$ de K.

Houve incremento da área foliar em todas as colheitas, contudo verificou-se que o maior incremento dessa variável, aproximadamente $150 \%$, aconteceu entre os 34 e 41 dias após o plantio de $M$. arvensis e de $M$. x piperita var. citrata; para $M$. x gracilis, isso ocorreu entre os 27 e 34 dias e representou quase $80 \%$, considerando a concentração de K de 276 $\mathrm{mg} \mathrm{L}^{-1}$. O período compreendido entre a primeira e terceira colheitas correspondeu à época em que as plantas investiram mais na formação de superfície foliar para captação de luz e conseqüentemente na produção e acúmulo de fotoassimilados.

Valmorbida (2003) verificou que os diferentes níveis de $\mathrm{K}$ na solução hidropônica de $M$. piperita não interferiram na área foliar, tendo observado aos 114 dias de idade maior valor de área foliar de 63,12 $\mathrm{dm}^{2}$. Em $M$. arvensis cultivada em vasos com capacidade de 6 L e solução nutritiva completa, Maia (1998a) obteve área foliar de 45,11 dm². Srivastava et al. (2003) constataram valor máximo de área foliar de $24,02 \mathrm{dm}^{2}$ para plantas de $M$. arvensis cv. Shivalik, cultivadas em solo, em casa de vegetação e colhidas aos 90 dias. Os resultados do presente trabalho foram bem superiores aos encontrados na literatura, devido às melhores condições experimentais.

Fitomassa fresca de folhas, de hastes e relação folha:haste. A fitomassa fresca de folhas e de hastes mostrouse significativa a partir dos 48 dias para $M$. arvensis (Figura 1c-d) e $M$. $\mathrm{x}$ piperita var. citrata (Figura 2c-d) com as doses estimadas de $\mathrm{K}$ para a máxima produção de folhas e de hastes, variando respectivamente de 397,2 a $416,6 \mathrm{mg} \mathrm{L}^{-1}$ e de 374,4 a 394,6 $\mathrm{mg} \mathrm{L}^{-1}(M$. arvensis); de 328,7 a 425,0 $\mathrm{mg} \mathrm{L}^{-1}$ e de 307,6 a 413,4 mg $\mathrm{L}^{-1}(M$. x piperita var. citrata). Neste estudo, o maior acúmulo de fitomassa fresca de folhas e de hastes do período experimental correspondeu respectivamente a 213,5 e $296,4 \mathrm{~g}(M$. arvensis) e a 328,9 e $373,1 \mathrm{~g}(M . \mathrm{x}$ piperita var. citrata). Em $M$. x gracilis (Figura 3c-d) tanto a fitomassa fresca de folhas como a de hastes apresentaram-se mais baixas nas plantas submetidas a concentrações de $\mathrm{K}$ maiores que $276 \mathrm{mg} \mathrm{L}^{-1}$, a partir dos 48 dias para folhas, e a partir dos 41 dias para hastes; aos 69 dias houve acúmulo máximo de 343,2 g de folhas e 324,1 g de hastes. Nessa espécie, a relação entre essas variáveis (Figura 3e) foi linear crescente 


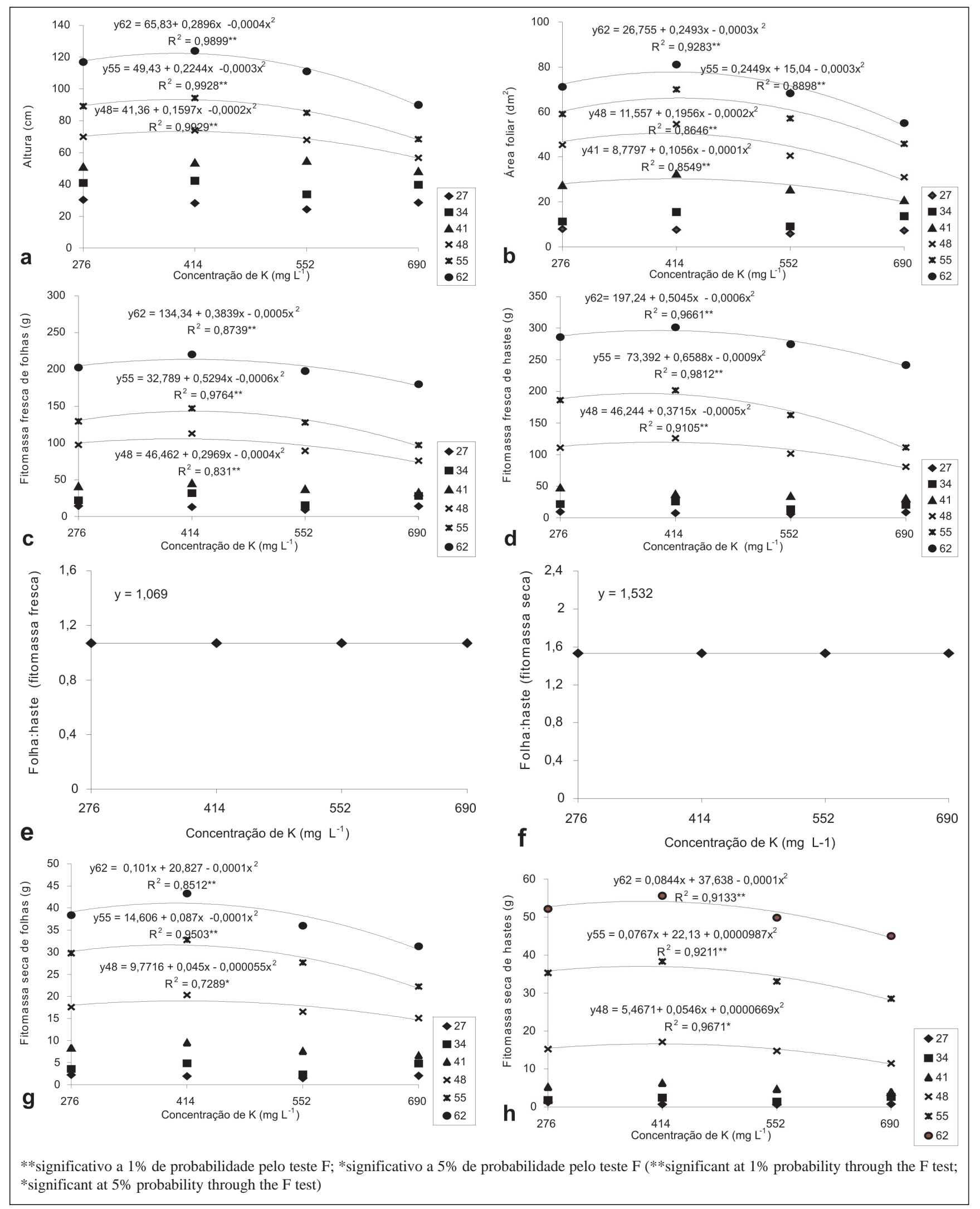

Figura 1. Altura (a), área foliar (b), fitomassa fresca de folhas (c), fitomassa fresca de hastes (d), folha:haste em fitomassa fresca (e), folha:haste em fitomassa seca (f), fitomassa seca de folhas (g), fitomassa seca de hastes (h) obtidos em função da concentração de K nas soluções hidropônicas e das idades de colheita (27; 34; 41; 48; 55; 62 dias) de Mentha arvensis (Height (a), leaves área (b), fresh leaves fitomass (c), fresh stem fitomass (d), leaf:stem relation of fresh fitomass (e), leaf:stem relation of dry fitomass (f), dry fitomass of leaves (g), dry fitomass of stem (h) obtained as a result of $\mathrm{K}$ concentration in the hidroponic solutions and date of harvest (27; 34; 41; 48; 55; 62 days) of Mentha arvensis). Santa Maria, UFSM, 2004. 
Tabela 1. Rendimento de fitomassa fresca de folhas, teor e rendimento do óleo essencial de três espécies de Mentha cultivadas em diferentes concentrações de K na solução hidropônica (yield of fresh fitomass of leaves, content and yield of essential oil of three Mentha species, cultivated in various concentrations of K in the hidroponic solution). Santa Maria, UFSM, 2004.

\begin{tabular}{|c|c|c|c|}
\hline \multirow{2}{*}{$\begin{array}{l}\text { Concentração de K } \\
\left(\mathrm{mg} \mathrm{L}^{-1}\right)\end{array}$} & \multirow{2}{*}{$\begin{array}{l}\text { Fitomassa fresca } \\
\left(\text { g planta }^{-1}\right)\end{array}$} & \multicolumn{2}{|c|}{ Óleo essencial* $^{*}$} \\
\hline & & Teor $(\%)$ & $\begin{array}{l}\text { Rendimento } \\
\text { (g planta-1) }^{-1} \text { ) }\end{array}$ \\
\hline \multicolumn{4}{|c|}{ Mentha arvensis $\mathrm{L}$. } \\
\hline 276 & 204,8 & 0,79 & 1,62 \\
\hline $412^{* *}$ & 213,5 & 0,88 & 1,88 \\
\hline 414 & 213,5 & 0,88 & 1,88 \\
\hline 552 & 204,4 & 0,98 & 2,00 \\
\hline 690 & 177,7 & 1,07 & 1,90 \\
\hline C.V. $(\%)$ & 7,32 & 9,61 & \\
\hline \multicolumn{4}{|c|}{ Mentha x piperita var. citrata (Ehrh.) Briq. } \\
\hline 276 & 322,5 & 0,76 & 2,45 \\
\hline $384^{* *}$ & 329,0 & 0,88 & 2,89 \\
\hline 414 & 328,5 & 0,91 & 2,99 \\
\hline 552 & 313,3 & 1,05 & 3,29 \\
\hline 690 & 276,8 & 1,20 & 3,32 \\
\hline C.V. $(\%)$ & 8,86 & 7,90 & \\
\hline \multicolumn{4}{|c|}{ Mentha x gracilis Solle } \\
\hline $276^{* *}$ & 343,2 & 0,83 & 2,85 \\
\hline 414 & 300,4 & 0,95 & 2,85 \\
\hline 552 & 257,6 & 1,07 & 2,76 \\
\hline 690 & 214,8 & 1,19 & 2,56 \\
\hline C.V. (\%) & 13,75 & 11,20 & \\
\hline
\end{tabular}

*Extraído a partir de amostras de $100 \mathrm{~g}$ de folhas frescas; **Dose estimada para obtenção da máxima produção de fitomassa fresca de folhas (*extracted of a $100 \mathrm{~g}$ sample of fresh leaves; **Evaluated doses to obtain the maximum yield of fresh leaves fitomass).

dos 41 até os 62 dias de idade, conforme o aumento na dose de $\mathrm{K}$, com valor superior a 1,0. Srivastava et al. (2003) estudaram a relação folha:haste em cultivares de $M$. arvensis, por 90 dias, em casa de vegetação e verificaram valores entre 1,1 e 1,5 indicando que houve maior partição de biomassa para folha do que para haste. Os resultados com $M$. x gracilis deste trabalho concordam com os desses autores, ressaltando-se que é desejável um aumento na relação folha:haste, pois a indústria retira o óleo essencial das folhas, sendo estas as partes da planta de maior valor comercial.

Embora as primeiras doses de $\mathrm{K}$ na solução nutritiva tenham aumentado a produção de fitomassa fresca de folhas e de hastes, em $M$. arvensis e $M$. x piperita var. citrata esse aumento foi proporcional entre as partes, pois a re- lação folha:haste em fitomassa fresca (Figura 1e, 2e) não foi influenciada pelo aumento da concentração do nutriente no meio, mantendo-se constante durante todo o período de desenvolvimento.

Verificou-se que, para as três espécies avaliadas, plantas mais altas produziram maior massa foliar, em função da idade. No entanto, na dose de K de $690 \mathrm{mg} \mathrm{L}^{-1}$ todas as plantas foram menores, ocorrendo também diminuição da massa de folhas.

Fitomassa seca de folhas, de hastes e relação folha:haste. A fitomassa seca, em $M$. arvensis, foi significativa dos 48 aos 62 dias, com as doses estimadas para a máxima produção de folhas (Figura 1g) variando entre 392,3 e 409,35 $\mathrm{mg} \mathrm{L}^{-1}$; e a de hastes (Figura 1h) entre 388,7 e 407,9 $\mathrm{mg} \mathrm{L}^{-1}$, havendo aos 62 dias acúmulo de 41,1 g de folhas e $54,2 \mathrm{~g}$ de hastes secas. Em $M$. x piperita var. citrata a fitomassa seca mostrou significância a partir dos 48 dias para folhas (Figura 2g) e a partir dos 55 dias para hastes (Figura 2h), variando a dose para máxima produção, respectivamente de 364,7 a $434,9 \mathrm{mg} \mathrm{L}^{-1}$ e de 328,2 a $396,5 \mathrm{mg} \mathrm{L}^{-1}$, acumulando aos 76 dias de idade $61,8 \mathrm{~g}$ de folhas e $67,1 \mathrm{~g}$ de hastes. Em M. x gracilis a fitomassa seca de folhas (Figura 3g) e a de hastes (Figura 3 h) foram significativamente mais baixas, a partir dos 48 dias, nas doses com maior concentração de $\mathrm{K}$, no entanto a melhor dose $\left(276 \mathrm{mg} \mathrm{L}^{-1}\right)$ proporcionou 74,6 $\mathrm{g}$ de folhas e 58,9 $\mathrm{g}$ de hastes. De maneira semelhante à fitomassa fresca, a relação entre essas variáveis secas (Figura 3f) foi significativa linearmente dos 41 aos 55 dias, aumentando conforme o aumento na dose de $\mathrm{K}$.

Da mesma forma como ocorreu com a relação folha:haste para fitomassa fresca, a relação de folha:haste para fitomassa seca, em M. arvensis (Figura 1f) e $M$. x piperita var. citrata (Figura 2f) não foi influenciada pelas concentrações crescentes de $\mathrm{K}$ no meio.

Segundo Duriyaprapan et al. (1986) em plantas de $M$. arvensis, cultivadas em câmara de crescimento, em vasos e colhidas aos 94 dias, houve rendimento de fitomassa seca de folhas de $13,1 \mathrm{~g} \mathrm{e}$ de hastes de 27,8 g, enquanto os resultados obtidos nesta pesquisa foram superiores aos desses autores.

\section{Óleo essencial}

O teor do óleo essencial das plantas foi afetado pela variação do $\mathrm{K}$ em todas as concentrações $(\mathrm{P}<0,01)$, conforme as respectivas equações de regressão ajustadas para cada espécie: $\mathrm{y}=0,6030+$ $0,000676 \mathrm{x}, \mathrm{R}^{2}=0,9400$ (M. arvensis); $\mathrm{y}=0,4684+0,0010638 \mathrm{x}, \mathrm{R}^{2}=0,9675$ (M. x piperita var. citrata) e $\mathrm{y}=0,5775$ $+0,0008909 \mathrm{x}, \mathrm{R}^{2}=0,9928(M . \mathrm{x}$ gracilis). $\mathrm{O}$ aumento na concentração de $\mathrm{K}$ promoveu aumento no teor do óleo para as três espécies de Mentha avaliadas (Tabela 1). As doses estimadas de $\mathrm{K}$ em mg L $\mathrm{L}^{-1}$ para ótima produtividade de folhas frescas, segundo equações de regressão das figuras $1 c, 2 c$ e $3 c$, correspondem a $412 \mathrm{mg} \mathrm{L}^{-1}(M$. arvensis), $384 \mathrm{mg} \mathrm{L}^{-1}$ (M. x piperita var. citrata) e $276 \mathrm{mg} \mathrm{L}^{-1}$ (M. x gracilis), 


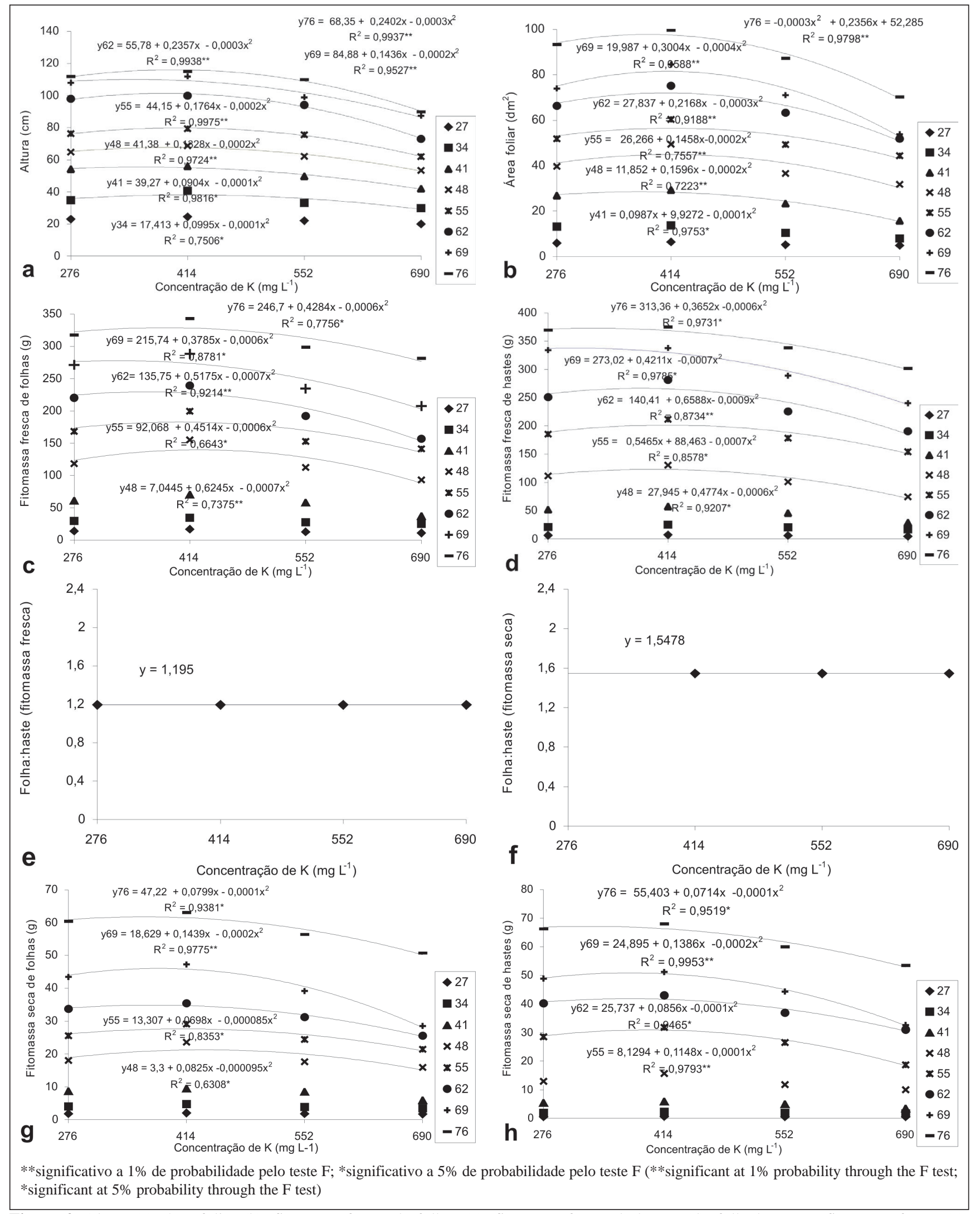

Figura 2. Altura (a), área foliar (b), fitomassa fresca de folhas (c), fitomassa fresca de hastes (d), folha:haste em fitomassa fresca (e), folha:haste em fitomassa seca (f), fitomassa seca de folhas (g), fitomassa seca de hastes (h) obtidos em função da concentração de K nas soluções hidropônicas e das idades de colheita (27; 34; 41; 48, 55, 62, 76 dias) de Mentha x piperita var. citrata (Height (a), leaves área (b), fresh leaves fitomass (c), fresh stem fitomass (d), leaf:stem relation of fresh fitomass (e), leaf:stem relation of dry fitomass (f), dry fitomass of leaves (g), dry fitomass of stem (h) obtained as a result of $\mathrm{K}$ concentration in the hidroponic solutions and date of harvest (27; 34; 41; 48; 55; 62 days) of Mentha x piperita var. citrata). Santa Maria, UFSM, 2004. 


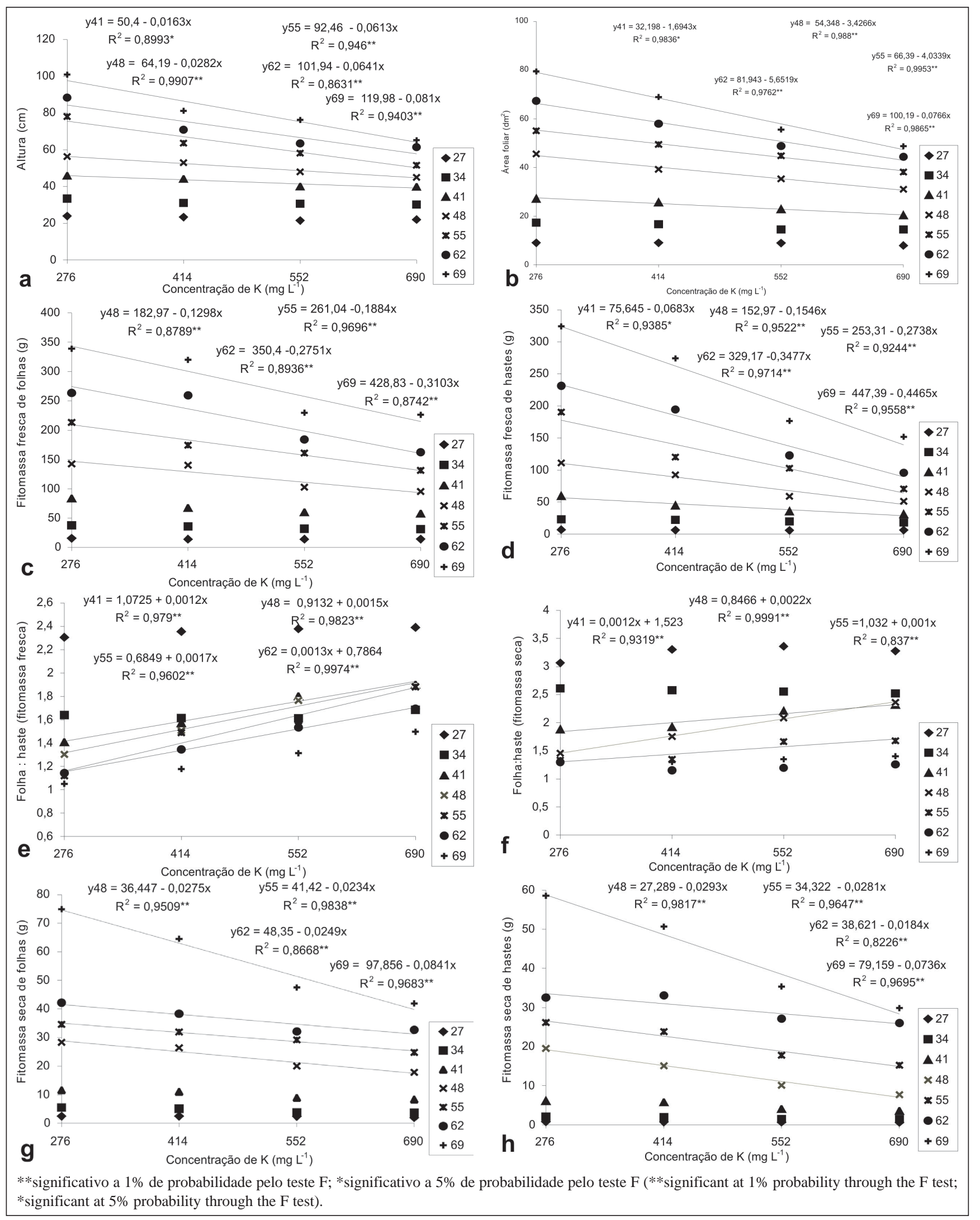

Figura 3. Altura (a), área foliar (b), fitomassa fresca de folhas (c), fitomassa fresca de hastes (d), folha:haste em fitomassa fresca (e), folha:haste em fitomassa seca (f), fitomassa seca de folhas (g), fitomassa seca de hastes (h) obtidos em função da concentração de K nas soluções hidropônicas e das idades de colheita (27; 34; 41; 48; 55; 62 e 69 dias) de Mentha x gracilis (Height (a), leaves área (b), fresh leaves fitomass (c), fresh stem fitomass (d), leaf:stem relation of fresh fitomass (e), leaf:stem relation of dry fitomass (f), dry fitomass of leaves $(\mathrm{g})$, dry fitomass of stem (h) obtained as a result of K concentration in the hidroponic solutions and date of harvest (27; 34; 41; 48; 55; 62 days) of Mentha $x$ gracilis). Santa Maria, UFSM, 2004. 
rendendo respectivamente em óleo essencial o equivalente a $1,88 \mathrm{~g}_{\text {planta }}{ }^{-1}$ $\left(188 \mathrm{~kg} \mathrm{ha}^{-1}\right) ; 2,89 \mathrm{~g} \mathrm{planta}^{-1}\left(289 \mathrm{~kg} \mathrm{ha}^{-1}\right)$ e $2,85 \mathrm{~g} \mathrm{planta}^{-1}\left(285 \mathrm{~kg} \mathrm{ha}^{-1}\right)$.

Um teor elevado de óleo por si só pode não ter interesse agrícola, pois plantas que produzam muito óleo, porém com baixa produção de folhas, resultarão em baixo rendimento de óleo por área. É o caso de $M$. x gracilis que apesar de suas folhas apresentarem maior teor de óleo na dose de K de 690 $\mathrm{mg} \mathrm{L}^{-1}$, a produção de folhas foi reduzida, resultando em menor rendimento de óleo por planta $\left(2,56 \mathrm{~g} \mathrm{planta}^{-1}\right)$ e seu equivalente por hectare $\left(256 \mathrm{~kg} \mathrm{ha}^{-1}\right)$. Por outro lado, observou-se que apenas em $M$. x piperita var. citrata a maior dose de $\mathrm{K}$ promoveu maior rendimento de óleo, isto é, $15 \%$ acima da dose ótima. Maia (1998b) em cultivo hidropônico de $M$. arvensis, colhida aos 68 dias após o plantio, obteve aumento significativo no teor de óleo essencial $(1,45 \%)$ e produção de óleo por vaso (1 $\mathrm{g} \mathrm{vaso}^{-1}$ ) na solução de $468 \mathrm{mg}$ de K L ${ }^{1}$, com incremento na produção de parte aérea. Todavia, em estudo com $M$. piperita aos 105 dias após transplante, Valmorbida (2003) encontrou rendimento de $147 \mathrm{~L} \mathrm{ha}^{-1}$ de óleo em plantas cultivadas em solução hidropônica com nível de $\mathrm{K}$ de 117/234 $\mathrm{mg} \mathrm{L}^{-1}$, porém com a diminuição na concentração de $\mathrm{K}$ para 58,50/117 $\mathrm{mg} \mathrm{L}^{-1}$, houve aumento no teor de óleo (176 L ha-1) e na fitomassa fresca $\left(66 \mathrm{t} \mathrm{ha}^{-1}\right)$, contrariando os resultados do presente trabalho.

A concentração de metabólitos secundários, segundo Tuomi et al. (1991), utilizados para defesa do vegetal tende a ter uma concentração inversa às taxas de crescimento, por ser este o "custo da defesa" das plantas, com o desvio de substâncias que poderiam gerar açúcares, proteínas e gorduras para produção de metabólitos secundários. Charles et al. (1990) constataram aumento linear no conteúdo de óleo por folha que foi de 44,0 para $70,8 \mu \mathrm{L} \mathrm{g}^{-1}$ de matéria seca, com diminuição exponencial de área foliar e biomassa de parte aérea, quando plantas de $M$. piperita foram submetidas a níveis aumentados de estresse osmótico. Embora no presente estudo não tenha sido medido o potencial osmótico das soluções, verificou-se redução no crescimento das plantas, provavelmente pelo excesso de aplicação de $\mathrm{KCl}$ no meio, conforme aumento da condutividade elétrica de 2,38 na solução padrão, para $3,87 \mathrm{mS} \mathrm{cm}^{-1}$ na solução com maior concentração de K. Esses mesmos autores ainda relatam que folhas produzidas por plantas cultivadas com altos níveis de estresse osmótico podem ter alta densidade de glândulas de óleo, como produto do estresse induzido pela redução da área foliar. E, além disso, citam que a redução no crescimento, induzida pelo mais baixo potencial osmótico pode ter resultado em novo padrão de partição de reservas, proporcionando esqueletos carbônicos para biossíntese e acumulação de terpenóides. Pelos valores obtidos neste trabalho supõe-se que também tenha havido aumento na quantidade de glândulas de óleo, concordando com as afirmações desses autores.

O valor máximo da concentração de $\mathrm{K}$, nas soluções hidropônicas, afeta negativamente o crescimento e a acumulação de fitomassa em plantas de Mentha, porém proporciona aumento no teor de óleo essencial por planta. Nas colheitas consecutivas observa-se aumento no crescimento das plantas das três espécies. As plantas de $M$. arvensis e $M$. x piperita var. citrata acumulam mais fitomassa de hastes do que de folhas, o contrário ocorrendo com $M$. x gracilis, que além disso apresenta maior produção equivalente de óleo por hectare, na concentração padrão de K. As concentrações de $\mathrm{K}$ sugeridas para o bom crescimento de Mentha, em cultivo hidropônico, encontram-se na faixa entre 276 e $414 \mathrm{mg} \mathrm{L}^{-1}$.

\section{REFERÊNCIAS}

BATAGLIA, OC. 2005. Métodos diagnósticos da nutrição potássica com ênfase no DRIS. In: YAMADA T; ROBERTS TL. SIMPÓSIO SOBRE POTÁSSIO NA AGRICULTURA BRASIlEIRA, 2, 2004, São Pedro, SP. Anais...Piracicaba: Associação Brasileira para Pesquisa da Potassa e do Fosfato.

BROWN B; HART J; WESCOTT MP; CHRISTENSEN NW. 2003. The critical role of nutrient management in mint production. Better Crops, 87: 9-11.
CASTELlANE PD.; ARAÚJO JAC. 1995. Cultivo sem solo - hidroponia. Jaboticabal: FUNEP. 43p.

CHARLES DJ; JOLY RL; SIMON JE. 1990. Effects of osmotic stress on the essential oil content and composition of peppermint. Phytochemistry 29:2837-2840.

DURIYAPRAPAN SB; BRITTEN EJ; BASFORD KE. 1986. The effect of temperature on growth, oil yield and oil quality of Japanese mint. Annals of Botany 58:729736.

LORENZI H; MATOS FJA. 2002. Plantas medicinais no Brasil: nativas e exóticas cultivadas. Nova Odessa, SP: Instituto Plantarum. p. 246251.

MAIA NB. 1998a. Efeito da nutrição mineral na qualidade do óleo essencial da menta (Mentha arvensis L.) cultivada em solução nutritiva. In: MING LC. Plantas Medicinais Aromáticas e condimentares: avanços na pesquisa agronômica. Botucatu: UNESP. p. 81-95.

MAIA, NB. 1998b. Produção e qualidade do óleo essencial de duas espécies de menta cultivadas em soluções nutritivas. Piracicaba: USPESALQ. 105p. (Tese doutorado).

MAIRAPETYAN SK. 1999. Aromatic plant culture in open-air hidroponics. Acta Horticulturae 502: 33-41.

MALAVOLTA E. 2006. Manual de nutrição mineral de plantas. São Paulo: Ceres. 638p.

MARSCHNER H. 1995. Mineral nutrition of higher plants. 2. ed. San Diego: Academic Press. 888p.

MARSCHNER H; CAKMAK I. 1989. High light intensity enhances chlorosis and necrosis in leaves of zink, potassium and magnesium deficient bean (Phaseolus vulgaris) plants. Journal of Plant Physiology 134:308-315.

PAULUS D; MEDEIROS SLP; SANTOS OS; MANFRON PA; DOURADO DN; BORCIONI E; FABBRIN E. 2004. Rendimento de biomassa e óleo essencial de menta japonesa (Mentha arvensis L.) Revista Brasileira de Plantas Medicinais 7: 34-42.

SANTOS OS (ed). 2000. Cultivo sem solo: hidroponia. Santa Maria: UFSM/CCR. 107p.

SINGH VP; CHATTERJEE BN; SINGH DV. 1989. Response of mint species to nitrogen fertilization. Journal of Agricultural Science 113:267-271.

SRIVASTAVA RK; SINGH AK; KALRA A; TOMAR VKS; BANSAL RP; PATRA DD; CHAND S; NAQVI AA; SHARMA S; KUMAR S. 2002. Characteristics of menthol mint Mentha arvensis cultivated on industrial scale in the Indo-Gangetic plains. Industrial crops and products 15: 189-198.

TUOMI J; FAGERSTRÖM T; NIEMELÃ P. 1991. Carbon allocation, phenotypic plasticity and induced defences. In: TALLAMY DW; RAUPP MJ. Phytochemical induction by herbivores. New York: John Wiley. p. 85-104.

VALMORBIDA J. 2003. Níveis de potássio em solução nutritiva, desenvolvimento de plantas e a produção de óleo essencial de Mentha piperita L. Botucatu: UNESP. 128p. (Tese mestrado). 